\title{
Bortezomib-based therapy for transplant-ineligible East Asian patients with newly diagnosed mantle- cell lymphoma
}

This article was published in the following Dove Press journal:

OncoTargets and Therapy

Jie Jin, ${ }^{1,2}$ Rumiko Okamoto, ${ }^{3}$

Sung-Soo Yoon, ${ }^{4}$ Lee-Yung

Shih, ${ }^{5}$ Jun Zhu, ${ }^{6}$ Ting Liu, ${ }^{7}$

Xiaonan Hong, ${ }^{8}$ Lixia Pei, 9

Brendan Rooney, ${ }^{10}$ Helgi van

de Velde, ${ }^{1}$ Huiqiang Huang ${ }^{12}$

'Department of Hematology, The First Affiliated Hospital of Zhejiang University College of Medicine, Hangzhou, Zhejiang, China; ${ }^{2}$ Key Laboratory of Hematologic Malignancies, Diagnosis and Treatment, Hangzhou, Zhejiang, China; ${ }^{3}$ Department of Chemotherapy, Tokyo Metropolitan Cancer and Infectious Diseases Center, Komagome Hospital, Tokyo, Japan; ${ }^{4}$ Department of Internal Medicine, Seoul National University College of Medicine, Seoul, Republic of Korea; ${ }^{5}$ Division of Hematology-Oncology, Chang Gung Memorial Hospital-Linkou, Chang Gung University, Taoyuan, Taiwan; ${ }^{6}$ Department of Lymphoma, Peking University Cancer Hospital \& Institute, Beijing, China; ${ }^{7}$ Division of Hematology, Department of Internal Medicine, West China Hospital, Sichuan University, Chengdu, Sichuan, China; ${ }^{8}$ Lymphoma and GI Medical Oncology, Fudan University Shanghai Cancer Center. Shanghai, China; 'Janssen Research \& Development, LLC, Raritan, NJ, USA; ${ }^{10}$ Janssen Research \& Development, High Wycombe, Buckinghamshire, UK; "Oncology Clinical Research, Millennium Pharmaceuticals, Inc., Boston, MA, USA ${ }^{12}$ Department of Medical Oncology,

Sun Yat-sen University Cancer Center, Guangzhou, Guangdong, China

Correspondence: Huiqiang Huang Ward 4, Internal Medicine, Sun Yat-sen University Cancer Center, 65I East Dongfeng Road, Guangzhou, Guangdong 510060, China

Tel/fax +86 2087343350

Email huang_sysu@163.com
Introduction: This subgroup analysis of the LYM-3002 Phase III study (NCT00722137) investigated whether substituting bortezomib for vincristine in frontline R-CHOP (rituximab plus cyclophosphamide, doxorubicin, vincristine, and prednisone) therapy could improve outcomes in East Asian patients with newly diagnosed mantle-cell lymphoma (MCL).

Materials and methods: A total of 121 East Asian patients from China, Taiwan, Japan, and the Republic of Korea with stage II-IV MCL who were ineligible or not considered for stem-cell transplantation were enrolled to six to eight 21-day cycles of R-CHOP or VR-CAP (R-CHOP with bortezomib replacing vincristine).

Results: The primary end point was progression-free survival. After a median follow-up of 42.4 months, median progression-free survival in East Asian patients was 13.9 (R-CHOP) versus 28.6 (VR-CAP) months (HR 0.7, $P=0.157$; 43\% improvement with VR-CAP). Secondary end points (R-CHOP vs VR-CAP), including complete response rate ( $47 \%$ vs $63 \%)$, duration of complete response (median 16.6 vs 46.7 months), and treatment-free interval (median 21 vs 46.5 months), were improved with VR-CAP. VR-CAP was associated with increased but manageable toxicity. The most frequent adverse events were hematologic toxicities.

Conclusion: VR-CAP was effective in East Asian patients with newly diagnosed MCL, and could be considered for patients in whom stem-cell transplantation is not an option.

Keywords: mantle-cell lymphoma, bortezomib, VR-CAP, R-CHOP

\section{Introduction}

Mantle-cell lymphoma (MCL) is an incurable and aggressive hematologic malignancy with poor prognosis (median overall survival [OS] 4-5 years). ${ }^{1,2} \mathrm{MCL}$ is a relatively rare form of B-cell lymphoma derived from mantle-zone B cells with a BCL1 translocation. MCL comprises 5\%-6\% of all non-Hodgkin lymphoma (NHL), worldwide. ${ }^{3}$ Of the NHL cases reported across East Asia, the frequency of MCL has been estimated at 4\% in Taiwan, ${ }^{4} 2.6 \%$ in China's Shanxi Province, ${ }^{5} 2.79 \%$ in Japan, ${ }^{6}$ and $1.5 \%$ in the Republic of Korea. ${ }^{7}$ These frequencies are lower than those reported in Europe $(4 \%-10 \%),{ }^{8-10}$ but similar to those observed in the US $(2.8 \%) .{ }^{11}$ A study of 21 male Taiwanese patients with MCL revealed that morphological variants identified in the Western population were also common to Taiwanese patients. ${ }^{12}$ The similarities in features of MCL between East Asian subpopulations and Western patients with MCL may indicate that the disease follows a similar course in both groups.

Complete response (CR) rates of up to $48 \%$ have been achieved using rituximab plus cyclophosphamide, doxorubicin, vincristine, and prednisone (R-CHOP), 
but progression-free survival (PFS) was limited (median 16.6 months). ${ }^{13}$ The efficacy of R-CHOP in East Asian patients with MCL is limited, derived primarily from retrospective studies. A Taiwanese single-institution analysis reported a 5-year OS rate of $52.7 \% .{ }^{4}$ A study of 18 Chinese patients with MCL who received R-CHOP therapy reported 2-year PFS and OS rates of 53\% and 59\%, respectively. ${ }^{14}$ In a Japanese study, 5-year OS was $62 \%$ in 64 patients with newly diagnosed MCL, of whom 41 received R-CHOP. ${ }^{15}$ In addition, a study of 501 Japanese patients with MCL who received rituximab-based therapy reported 5-year OS rates of $74 \%, 70 \%$, and $35 \%$ in patients with low-, intermediate-, and high-risk International Prognostic Index (IPI) scores, respectively. ${ }^{16}$

For patients ineligible or not considered for intensive chemotherapy and stem-cell transplantation, R-CHOP has until recently been considered the standard frontline therapy. ${ }^{17-20}$ As a result of the LYM-3002 Phase III trial (NCT00722137), the proteasome inhibitor bortezomib is approved for the treatment of both frontline and relapsed MCL in the US ${ }^{21}$ and 53 other countries. The LYM-3002 study was performed to investigate the benefit of substituting bortezomib for vincristine (VR-CAP), due to concerns over potential overlapping neurotoxicity ${ }^{22}$ and determine whether the combination could improve outcomes in stem-cell transplant-ineligible patients with MCL. ${ }^{20}$ This study identified that median PFS after a median follow-up of 40 months was 24.7 versus 14.4 months with VR-CAP and R-CHOP, respectively (HR 0.63, $P<0.001 ; 59 \%$ improvement with VR-CAP). VR-CAP was more efficacious than R-CHOP in terms of $\mathrm{CR}$ rate ( $53 \%$ vs $42 \%$ ), median duration of $\mathrm{CR}$ (DOCR; 42.1 vs 18 months), treatment-free interval (TFI; 40.6 vs 20.5 months) and 4-year OS rates (64\% vs 54\%). Experience in multiple myeloma has shown a similar safety profile between Asian and Western patients who received bortezomib. ${ }^{23-26}$ However, some unique adverse event (AE) profiles have been described with bortezomib in Japan, ${ }^{27,28}$ suggestive of differences in pharmacogenomics between Asian and Western multiple-myeloma populations.

At present, there have been no studies specifically evaluating the efficacy and safety of bortezomib in East Asian patients with MCL. The efficacy, safety, and exposure profiles of oncology drugs can differ greatly between Western and Asian patients (eg, resulting in regional differences in doses), ${ }^{29}$ highlighting the importance of determining treatment effects in the Asian population. This post hoc retrospective subgroup analysis thus evaluated the efficacy and safety of VR-CAP versus R-CHOP in East Asian patients (defined as those enrolled from China, Japan, Republic of Korea, and Taiwan) with newly diagnosed MCL who were ineligible for stem-cell transplantation, who were enrolled into the LYM-3002 trial.

\section{Materials and methods \\ Patients and study design}

Inclusion/exclusion criteria for the LYM-3002 study have been described previously. ${ }^{20}$ Briefly, adults with newly diagnosed stage II-IV MCL who were ineligible or not considered for stem-cell transplantation were enrolled between May 2008 and December 2011. Patient diagnosis was confirmed by central pathology review. The trial was conducted according to the Declaration of Helsinki, Good Clinical Practice guidelines, and local regulatory requirements. The trial protocol was approved by local ethics committees/institutional review boards. All patients provided written informed consent prior to the commencement of the study.

Patients were randomized 1:1 to receive six 21-day cycles of VR-CAP or R-CHOP (up to eight cycles if response first documented at cycle six). VR-CAP consisted of rituximab $375 \mathrm{mg} / \mathrm{m}^{2}$ intravenously (IV) on day 1 , cyclophosphamide $750 \mathrm{mg} / \mathrm{m}^{2} \mathrm{IV}$ on day 1 , doxorubicin $50 \mathrm{mg} / \mathrm{m}^{2} \mathrm{IV}$ on day 1 , prednisone $100 \mathrm{mg} / \mathrm{m}^{2}$ orally on days $1-5$, and bortezomib $1.3 \mathrm{mg} / \mathrm{m}^{2}$ IV on days $1,4,8$, and 11 . In the R-CHOP arm, the same regimen was used, except bortezomib was replaced by vincristine $1.4 \mathrm{mg} / \mathrm{m}^{2} \mathrm{IV}$ on day 1 only (maximum total dose $2 \mathrm{mg}$ ). Patients were stratified by their IPI score (low [0-1], low-intermediate [2], high-intermediate [3], and high [4-5]) and disease stage (American Joint Committee on Cancer NHL staging system) at diagnosis (II-IV).

\section{Assessments}

The primary end point was PFS, based on blinded independent radiology review committee (IRRC) assessment of disease progression (for the list of IRRCs and ethics committees please see the Table S1). PFS per investigator assessment was also evaluated and used as supportive evidence. Secondary end points were $\mathrm{CR}$ plus unconfirmed $\mathrm{CR}(\mathrm{CRu})$ rate, overall response rate (ORR; $\mathrm{CR} / \mathrm{CRu}$ plus partial response), time to response, duration of response (DOR), DOCR/CRu, time to progression (TTP), time to next (antilymphoma) therapy (TTNT), TFI, OS, and safety.

Computed tomography (CT) scans were performed every two cycles (6 weeks) during treatment and every 6-8 weeks during follow-up until disease progression, study discontinuation, initiation of alternate therapy, or death. At enrollment, up to ten measurable sites of disease (clearly 
measurable in two perpendicular dimensions: $>1.5 \mathrm{~cm}$ in the long axis, $>1 \mathrm{~cm}$ in the short axis) were measured using CT scanning and tracked. At each subsequent assessment, the sum of the product of the diameters of the measurable sites was calculated (ie, the sum of the long axis and short axis of all tracked lesions). All CT results were reviewed according to modified International Workshop to Standardize Response Criteria for NHL. ${ }^{30}$ AEs were graded using the National Cancer Institute's Common Terminology Criteria for Adverse Events version 3.0. ${ }^{31}$

\section{Statistical analyses}

In this post hoc subgroup analysis, all primary and secondary efficacy assessments were performed on the East Asian intent-to-treat subpopulation (containing all randomized subjects from Japan, the Republic of Korea, China, and Taiwan), except for response end points (which were analyzed in the response-evaluable population) and TFI data (analyzed in the safety population). The response-evaluable population was defined as all patients in the intent-to-treat population who received at least one dose of the study drug, had at least one measurable tumor mass at baseline, and had at least one postbaseline tumor assessment by IRRC, before any subsequent antilymphoma treatment. The safety population was defined as all randomized patients who received at least one dose of study drug.

The database lock for these analyses was January 10, 2014. Kaplan-Meier methodology was used to estimate timeto-event distributions, with stratified log-rank tests and Cox proportional-hazard models ( $\alpha=0.05$, two-sided) used for interarm comparisons of time-to-event end points. A stratified Cochran-Mantel-Haenszel $\chi^{2}$ test with IPI score and disease stage at diagnosis as stratification factors was used to assess interarm differences in response rates. Of note, the study was not powered to determine the advantages of VR-CAP over $\mathrm{R}-\mathrm{CHOP}$ in the East Asian population.

\section{Results \\ Patients}

In the LYM-3002 Phase III study, a total of 121 East Asian patients were enrolled across ten centers in Japan, two in the Republic of Korea, ten in China, and one in Taiwan. Of these, 73 were randomized to VR-CAP and 48 to R-CHOP (Figure S1); 18 patients were enrolled in Japan (VR-CAP, $\mathrm{n}=7$; R-CHOP, $\mathrm{n}=11$ ), five in the Republic of Korea (VRCAP, $n=3$; R-CHOP, $n=2$ ), 95 in China (VR-CAP, $n=61$; R-CHOP, $n=34$ ), and three in Taiwan (VR-CAP, $n=2$; R-CHOP, $n=1$ ).
In the East Asian population, patient demographic and baseline characteristics were generally well balanced between the two groups (Table 1), with similar median ages of 64 and 62 years in the VR-CAP and R-CHOP groups, respectively. Lymphoma was identified in the bone marrow of $49 \%$ and $58 \%$ of VR-CAP and R-CHOP patients, respectively. Treatment exposure was similar between groups (Table 2). The median number of cycles received was six, with $85 \%$ of patients receiving six or more cycles in both the VR-CAP and R-CHOP groups. For drugs common to both regimens, mean relative dose intensity (proportion of dose prescribed that was actually received) was $\geq 91 \%$ across both groups. In the VR-CAP group, the mean relative dose intensity of bortezomib was $78 \%$, and in the R-CHOP group the mean relative dose intensity for vincristine was $86 \%$.

\section{Efficacy}

After a median follow-up of 42.4 (range 0-63.3) months (VR-CAP 42.4 [range 0-60.8] months, R-CHOP 42.9 [range 0.8-63.3] months), and with 40 (55\%) and 36 (75\%)

Table I Patient demographics and baseline characteristics (intentto-treat population)

\begin{tabular}{|c|c|c|}
\hline Variable & $\begin{array}{l}\text { VR-CAP } \\
(n=73)\end{array}$ & $\begin{array}{l}\text { R-CHOP } \\
(n=48)\end{array}$ \\
\hline \multicolumn{3}{|l|}{ Age, years } \\
\hline Median (range) & $64(26-76)$ & $62(34-82)$ \\
\hline Male, n (\%) & $56(77)$ & $38(79)$ \\
\hline \multicolumn{3}{|l|}{ Disease stage at diagnosis, $\mathrm{n}(\%)^{\mathrm{a}}$} \\
\hline II & $5(7)$ & $4(8)$ \\
\hline III & $26(36)$ & $16(33)$ \\
\hline IV & $42(58)$ & $28(58)$ \\
\hline \multicolumn{3}{|l|}{ ECOG performance status, $n(\%)^{a}$} \\
\hline 0 & $46(63)$ & $30(63)$ \\
\hline I & $23(32)$ & $15(31)$ \\
\hline 2 & $4(6)$ & $3(6)$ \\
\hline \multicolumn{3}{|l|}{ IPI score (risk category), n (\%), a,b } \\
\hline $0-1$ (low) & $17(23)$ & $12(25)$ \\
\hline 2 (low-intermediate) & $27(37)$ & $15(3 \mid)$ \\
\hline 3 (high-intermediate) & $22(30)$ & $15(31)$ \\
\hline $4-5$ (high) & $7(10)$ & $6(13)$ \\
\hline Elevated LDH, n (\%) & $18(25)$ & $12(25)$ \\
\hline Bone-marrow involvement, n (\%) & $36(49)$ & $28(58)$ \\
\hline \multicolumn{3}{|l|}{ Reason for transplant ineligibility, $n(\%)^{c}$} \\
\hline Age & $51(70)$ & $30(63)$ \\
\hline Assessed as unable to tolerate HDT & $3(4)$ & $3(4)$ \\
\hline Comorbidity & $2(3)$ & $2(4)$ \\
\hline Other & $20(27)$ & $16(33)$ \\
\hline
\end{tabular}

Notes: ${ }^{\text {aSum }}$ of percentages may not equal 100\%, due to rounding; bdata from stratification; 'as assessed by Investigators.

Abbreviations: ECOG, Eastern Cooperative Oncology Group; HDT, high-dose therapy; IPI, International Prognostic Index; LDH, lactate dehydrogenase; R-CHOP, rituximab + cyclophosphamide, doxorubicin, vincristine, prednisone; VR-CAP, bortezomib, rituximab, cyclophosphamide, doxorubicin, prednisone. 
Table 2 Treatment exposure

\begin{tabular}{|c|c|c|}
\hline Variable & $\begin{array}{l}\text { VR-CAP } \\
(n=72)\end{array}$ & $\begin{array}{l}\text { R-CHOP } \\
(n=48)\end{array}$ \\
\hline \multicolumn{3}{|l|}{ Treatment cycles received } \\
\hline Median & 6 & 6 \\
\hline Range & $2-8$ & $\mathrm{I}-8$ \\
\hline Received $\geq 6$ cycles, $n(\%)^{a}$ & $61(85)$ & $4 \mid(85)$ \\
\hline Received 8 cycles, n (\%) & $3(4)$ & $3(6)$ \\
\hline \multicolumn{3}{|l|}{ Treatment duration, weeks } \\
\hline Median & 17.7 & 16.6 \\
\hline Range & $0.1-26.6$ & $0.1-32.7$ \\
\hline Completed treatment, $\mathrm{n}(\%)^{\mathrm{b}}$ & $60(83)$ & $4 I(85)$ \\
\hline Discontinued treatment, $\mathrm{n}(\%)^{\mathrm{b}}$ & $12(17)$ & $7(15)$ \\
\hline \multicolumn{3}{|c|}{ Relative dose intensity, ${ }^{c}$ mean percentage (SD) } \\
\hline Rituximab & $100(1)$ & $100(0)$ \\
\hline Cyclophosphamide & $91(11)$ & $97(7)$ \\
\hline Doxorubicin & $95(\mathrm{II})$ & $97(9)$ \\
\hline Prednisone & $92(16)$ & $93(16)$ \\
\hline Vincristine $/$ bortezomib & $78(14)$ & $86(9)$ \\
\hline
\end{tabular}

Notes: ${ }^{a}$ Values represent the number of patients who received one or more component of each therapy in one or more cycles; bsum of percentages may not equal $100 \%$, due to number rounding; 'dose received as a proportion of the dose prescribed; 'total vincristine dose capped at a maximum of $2 \mathrm{mg}$.

Abbreviations: R-CHOP, rituximab + cyclophosphamide, doxorubicin, vincristine, prednisone; VR-CAP, bortezomib, rituximab, cyclophosphamide, doxorubicin, prednisone.

progression/death events in the VR-CAP and R-CHOP arms, respectively, median PFS by IRRC was 28.6 months (VRCAP) versus 13.9 months (R-CHOP) (HR 0.7, $P=0.157$ ) (Figure 1A), representing a 30\% reduction in the risk of disease progression or death with VR-CAP. Median PFS by investigator assessment was 27.7 months (VR-CAP) versus 16.1 months (R-CHOP) (HR 0.58, $P=0.03$ ) (Figure 1B).

Of the 121 patients enrolled in East Asia, 115 patients (70 VR-CAP, 45 R-CHOP) were evaluable for response (Table 3). By IRRC assessment, ORRs were $97 \%$ and $98 \%$ for VR-CAP and R-CHOP, respectively, and rates of CR/ $\mathrm{CRu}$ (verified by bone marrow and lactate dehydrogenase) were $63 \%$ for the VR-CAP group and $47 \%$ for R-CHOP. Median time to response was 1.4 months in both arms. Median DOR with VR-CAP and R-CHOP was 30.7 and 14.4 months, respectively, and respective median durations of $\mathrm{CR} / \mathrm{CRu}$ were 46.7 and 16.6 months. Median TTP by IRRC was 30.9 versus 15.7 months with VR-CAP versus R-CHOP. Investigator assessments of response were consistent with the IRRC assessments (Table 3).

At data cutoff, 21 (29\%) VR-CAP patients and 28 (58\%) R-CHOP patients had received subsequent antilymphoma therapy following their respective frontline treatments. The types of subsequent therapy received in second-line therapy and beyond were generally similar across the two arms. Of note, 0 patients in the VR-CAP arm and seven (15\%) in the R-CHOP arm received subsequent bortezomib. Median TTNT was 50.8 versus 24.8 months (Figure 2A) and median TFI 46.5 versus 21 months with VR-CAP versus R-CHOP.

OS data were not mature at data cutoff, with 37 (31\%) deaths in the East Asian population (22 [30\%] in the VR-CAP arm and 15 [31\%] in the R-CHOP arm). Median OS was not reached with VR-CAP versus 56.3 months with R-CHOP (Figure 2B). The 4-year survival rates were $62 \%$ in the VR-CAP group and $61 \%$ in the R-CHOP group.

\section{Safety}

Of the 121 East Asian patients who were randomized, 120 received at least one dose of the study drug and were included in the safety population. One patient was excluded from the VR-CAP arm, because they chose to withdraw following randomization prior to receiving any study treatment. All patients in both groups experienced at least one treatmentemergent AE. The most common AEs are summarized in Table 4; AEs with a $\geq 20 \%$ difference between groups were thrombocytopenia (VR-CAP 99\%, R-CHOP 35\%), anemia ( $81 \%$ vs $58 \%$ ), lymphopenia ( $64 \%$ vs $42 \%$ ), diarrhea ( $47 \%$ vs $10 \%$ ), pyrexia ( $47 \%$ vs $23 \%$ ), and nausea ( $31 \%$ vs $8 \%$ ).

Grade $\geq 3$ treatment-emergent AE rates were $100 \%$ in the VR-CAP arm versus $94 \%$ in the R-CHOP arm. The most frequent grade $\geq 3$ AEs with $\geq 10 \%$ difference between arms were hematologic toxicities, including neutropenia (VR-CAP 97\%, R-CHOP 79\%), leukopenia ( $89 \%$ vs 54\%), thrombocytopenia ( $83 \%$ vs $13 \%$ ), and lymphopenia ( $60 \%$ vs $33 \%$ ). Diarrhea was the most common grade $\geq 3$ nonhematologic toxicity (VR-CAP 14\%, R-CHOP 4\%) (Table 4). Grade $\geq 4$ AE rates were $83 \%$ in the VR-CAP arm versus $69 \%$ in the $\mathrm{R}$-CHOP arm. The most common grade $\geq 4$ AEs, with a frequency of $\geq 5 \%$ in either group, were hematologic toxicities, including thrombocytopenia (VR-CAP 51\%, R-CHOP $4 \%$ ), leukopenia (64\% vs $25 \%$ ), and lymphopenia (38\% vs $4 \%$ ). In the VR-CAP and R-CHOP groups, respectively, four $(6 \%)$ and five $(10 \%)$ patients discontinued due to AEs, and six (8\%) and two (4\%) had grade 5 AEs. Two patients in the VR-CAP arm and one in the R-CHOP group died during the study due to AEs: in the VR-CAP arm, one patient had left ventricular dysfunction and aspiration that was considered as possibly related to treatment, and one had aspiration pneumonia that was considered unrelated to study therapy; in the R-CHOP arm, one patient had cardiac failure that was considered as being related to study treatment (most likely to doxorubicin).

The incidence of serious AEs was 26\% with VR-CAP and $27 \%$ with R-CHOP. Rates of individual serious AEs 

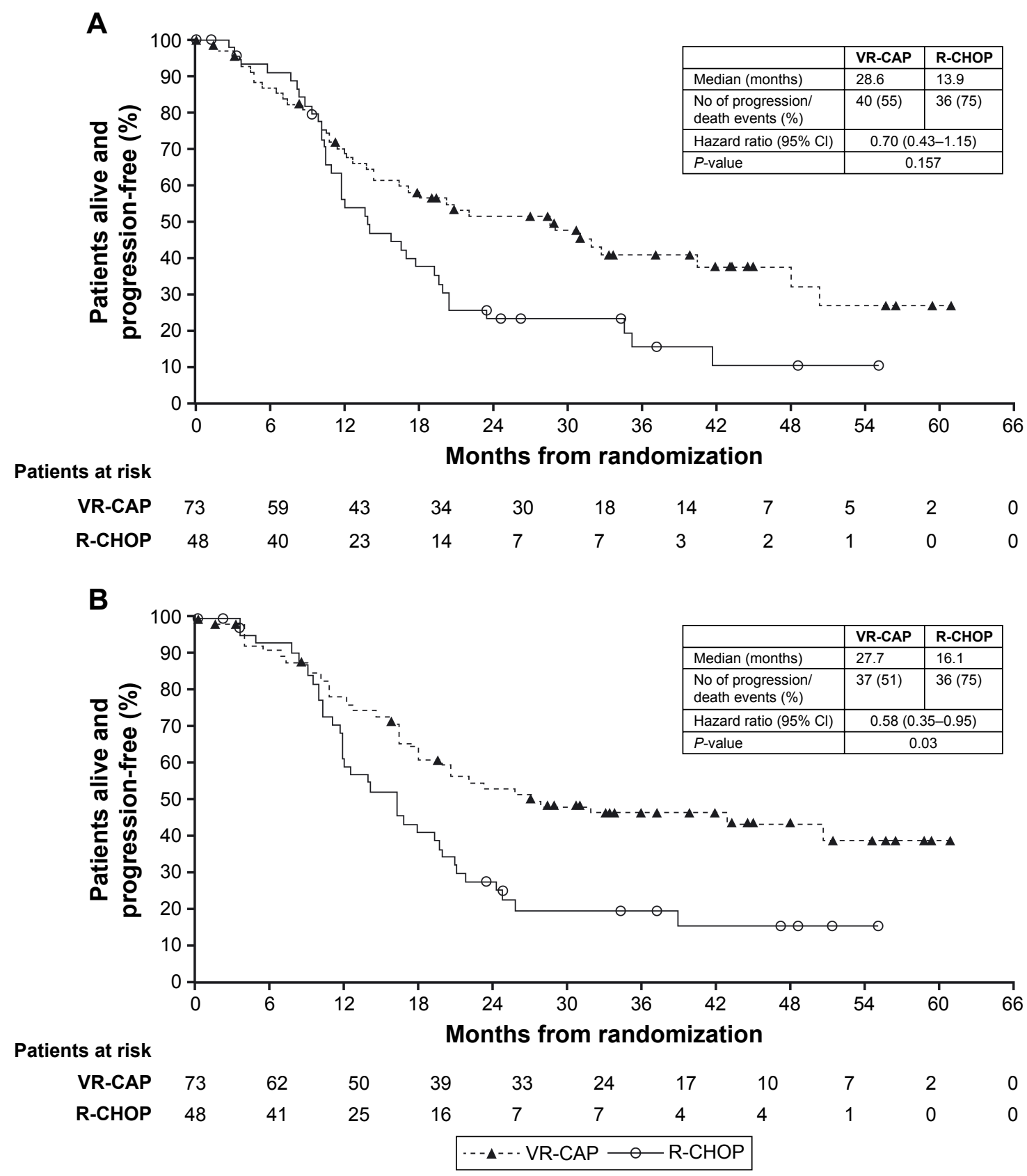

Figure I Kaplan-Meier analysis of progression-free survival in the East Asian population.

Notes: (A) Independent Radiology Review Committee; (B) investigator assessment.

Abbreviations: R-CHOP, rituximab + cyclophosphamide, doxorubicin, vincristine, prednisone; VR-CAP, bortezomib, rituximab, cyclophosphamide, doxorubicin, prednisone.

were $<5 \%$ in each treatment group, except for febrile neutropenia (VR-CAP 8\%, R-CHOP 4\%), neutropenia (VR-CAP 8\%, R-CHOP 6\%), thrombocytopenia (VR-CAP $6 \%$, R-CHOP 0\%), pneumonia (VR-CAP 3\%, R-CHOP 6\%), and pyrexia (VR-CAP 6\%, R-CHOP 2\%).

The management of key AEs - thrombocytopenia, neutropenia, and peripheral neuropathy $(\mathrm{PN})$ - in both groups is summarized in Table 5. The rates of red blood cell (RBC) transfusions were $15 \%$ with VR-CAP and 13\% with R-CHOP, and platelet transfusions with VR-CAP were $35 \%$ and $4 \%$ with R-CHOP. Rates of any-grade bleeding events were 3\% for VR-CAP and $2 \%$ for R-CHOP. In total, $96 \%$ of patients in the VR-CAP arm and $85 \%$ in the R-CHOP arm received colony-stimulating-factor therapy before or during study treatment; in 19 and nine patients, respectively, this was employed prophylactically. Reactivation of hepatitis B virus (HBV) was similar between groups $-3 \%$ versus $2 \%$. There were $15 \mathrm{PN}$ events in the VR-CAP group and 14 in the R-CHOP group. 
Table 3 Response rates in VR-CAP versus R-CHOP arms by Independent Radiology Review Committee and investigator assessment

\begin{tabular}{|c|c|c|c|c|}
\hline \multirow[t]{2}{*}{ End point } & \multicolumn{2}{|l|}{ IRRC } & \multicolumn{2}{|l|}{ IA } \\
\hline & VR-CAP & R-CHOP & VR-CAP & R-CHOP \\
\hline Best response rate & $\mathrm{n}=70$ & $n=45$ & $\mathrm{n}=70$ & $\mathrm{n}=45$ \\
\hline Overall response, $n(\%)^{\mathrm{a}}$ & $68(97)$ & $44(98)$ & $66(94)$ & $44(98)$ \\
\hline Complete response, n (\%) & $44(63)$ & $21(47)$ & $29(4 I)$ & $13(29)$ \\
\hline Time to response & $\mathrm{n}=70$ & $\mathrm{n}=45$ & $n=70$ & $\mathrm{n}=45$ \\
\hline Median, months & 1.4 & 1.4 & 1.4 & 1.4 \\
\hline Duration of response & $n=68$ & $\mathrm{n}=44$ & $\mathrm{n}=66$ & $\mathrm{n}=44$ \\
\hline Median, months $(95 \% \mathrm{Cl})$ & $30.7(16.9-49.1)$ & $14.4(9-18)$ & 41.4 (19.5-NE) & $14.8(9.5-19.5)$ \\
\hline Duration of complete response & $\mathrm{n}=44$ & $\mathrm{n}=21$ & $\mathrm{n}=29$ & $\mathrm{n}=13$ \\
\hline Median, months $(95 \% \mathrm{Cl})$ & 46.7 (27.2-NE) & $16.6(9.7-33.9)$ & NE (48-NE) & 19.3 (7.6-NE) \\
\hline Time to progression & $\mathrm{n}=73$ & $\mathrm{n}=48$ & $\mathrm{n}=73$ & $\mathrm{n}=48$ \\
\hline Median, months $(95 \% \mathrm{Cl})$ & $30.9(17.1-50.2)$ & $15.7(\mid 1.7-19.5)$ & 42.7 (20.4-NE) & $16.2($ (I I.7-20.7) \\
\hline
\end{tabular}

Notes: ${ }^{a}$ Complete response plus unconfirmed complete response, confirmed by evidence of bone-marrow clearance and LDH normalization. ${ }^{b} \mathrm{Complete}$ response plus unconfirmed complete response.

Abbreviations: Cl, confidence interval; IRRC, Independent Radiology Review Committee; IA, investigator assessment; NE, not estimable; R-CHOP, rituximab + cyclophosphamide, doxorubicin, vincristine, prednisone; VR-CAP, bortezomib, rituximab, cyclophosphamide, doxorubicin, prednisone.

Complete resolution of PN was achieved in $73 \%$ versus $79 \%$ of events in a median time of 11.8 versus 8.7 months for VRCAP and R-CHOP, respectively (Table 5).

\section{Discussion}

This subgroup analysis of the large, international, randomized Phase III LYM-3002 study was the first to evaluate the clinical benefit of bortezomib-based therapy in East Asian patients with newly diagnosed MCL. A 43\% improvement in median PFS was observed in East Asian patients who received VR-CAP compared with R-CHOP (HR 0.7, $P=0.157$ ), a finding that was consistent with the benefit observed in the overall LYM-3002 population (HR 0.63). ${ }^{20}$ Also consistent with the findings in the overall population, VR-CAP was associated with increased, yet manageable, toxicity in East Asian patients. Despite these similarities, the retrospective nature of this subgroup analysis means that it was not powered to determine a statistically significant difference in PFS between VR-CAP and R-CHOP in the East Asian subgroup. The analysis was limited by the size of the East Asian subpopulation and the number of PFS events that had occurred at data cutoff. Because of this, the HR was the most valuable metric, providing a measure of the treatment effect of VR-CAP versus R-CHOP in the East Asian subgroup. Nevertheless, these findings suggest that VR-CAP is a feasible and active treatment option in East Asian patients with MCL who are ineligible for transplant.

The primary end point data on PFS are supported by findings for the secondary efficacy end points. Clinically relevant improvements included higher rates of $\mathrm{CR} / \mathrm{CRu}$ in the VR-CAP group, which were more durable than those observed with R-CHOP. The CR/CRu rates (63\% [VR-CAP] and 47\% [R-CHOP]) and DOCR (46.7 and 16.6 months, respectively) in the East Asian population were similar to those observed in the overall population (CR/Cru 53\% [VRCAP] and 42\% [R-CHOP], DOCR 42.1 versus 18 months, respectively)..$^{20}$ Additionally, there was a doubling of TTP and TTNT in VR-CAP-treated patients compared with those receiving R-CHOP. TTP was 1.3 years longer in the VR-CAP group compared with the R-CHOP group, and TTNT was extended by 2.2 years in the VR-CAP group. Compared with the overall LYM-3002 population, both TTP and TTNT for East Asian patients were lower, irrespective of the treatment group. Based on median values, VR-CAP patients also benefited from an additional 2.1-year treatment-free period. OS data are not yet mature, with only $31 \%$ of patients in the East Asian subgroup having died after a median follow-up period of 39.4 and 40.3 months in the VR-CAP and R-CHOP arms, respectively, resulting in minimal difference between VR-CAP and R-CHOP in terms of 4-year survival rates. This contrasts with the overall study data, in which VR-CAP provided a 4-year survival advantage over R-CHOP of $10 \%$, determined after a similar percentage of patients had died (32\%, median duration of follow-up 38.9 months). These results should be interpreted with caution, due to the smaller sample size of the East Asian subgroup versus the overall population.

At the time of subgroup analysis, there were limited data available for the safety of R-CHOP- and bortezomib-based therapies in an East Asian population. This was the first analysis performed to evaluate the use of bortezomib for the treatment of newly diagnosed MCL in this population. 


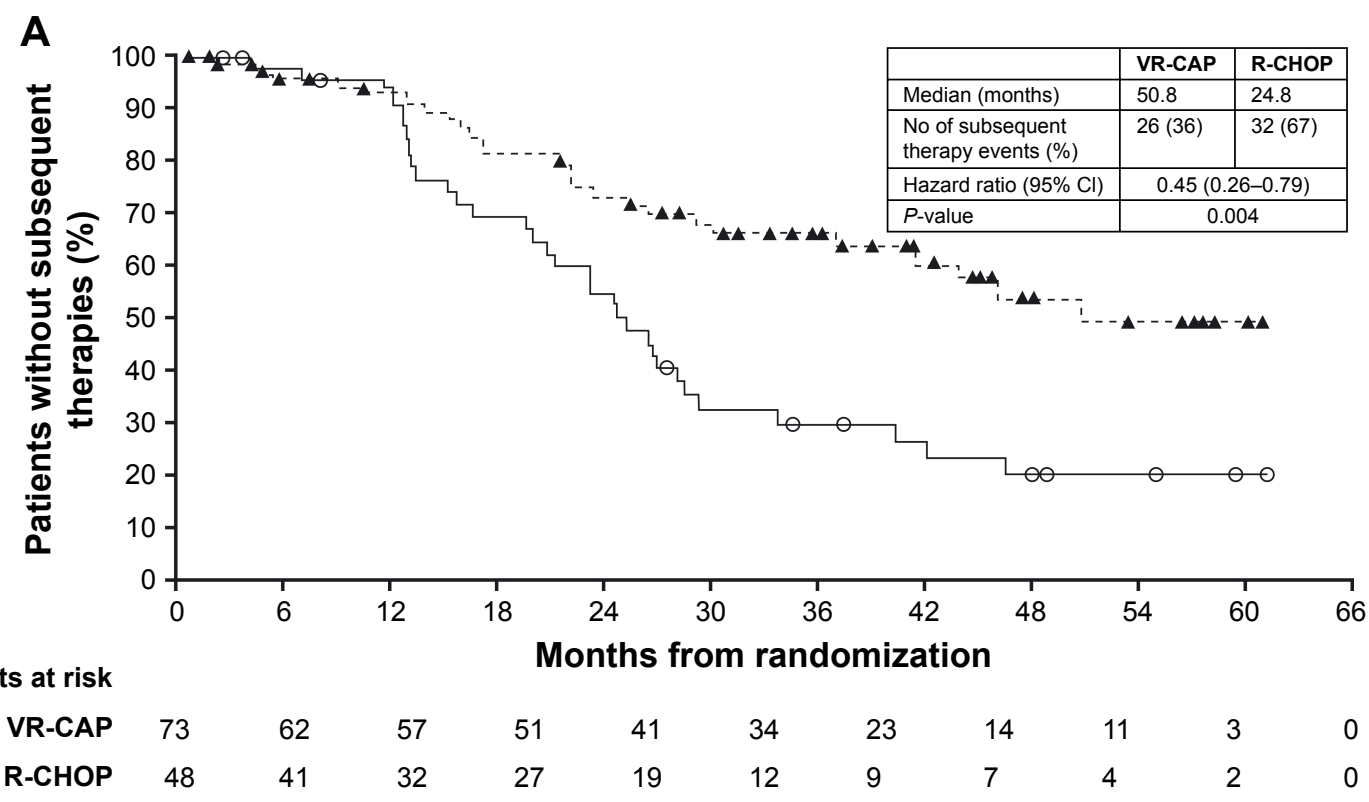

\begin{tabular}{|c|c|c|c|c|c|c|c|c|c|c|}
\hline Patients at risk & & & & & 110 & 180 & min & & & \\
\hline VR-CAP & 73 & 62 & 57 & 51 & 41 & 34 & 23 & 14 & 11 & 3 \\
\hline R-CHOP & 48 & 41 & 32 & 27 & 19 & 12 & 9 & 7 & 4 & 2 \\
\hline
\end{tabular}

B

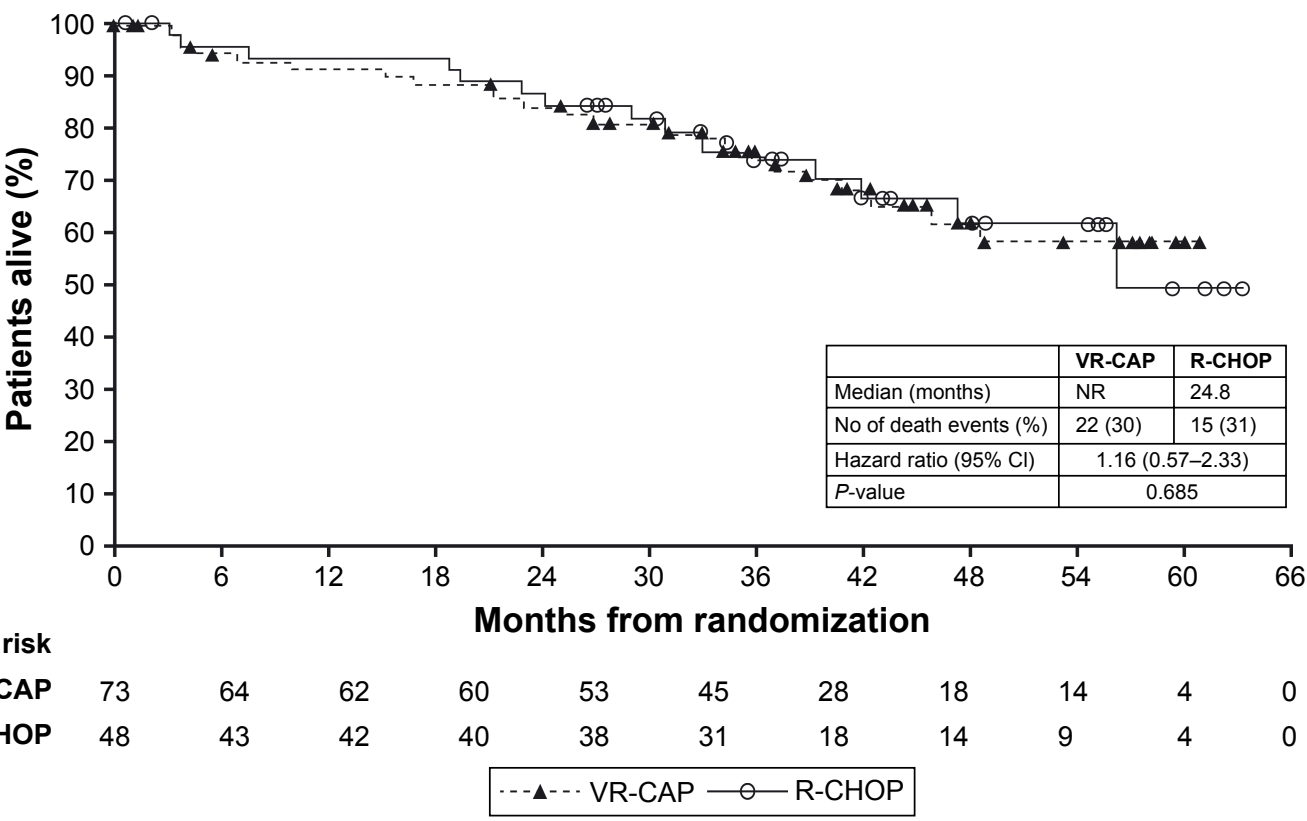

Figure 2 Kaplan-Meier analysis of (A) time to next antilymphoma therapy and (B) overall survival, in the East Asian population (intent-to-treat population).

Abbreviations: R-CHOP, rituximab + cyclophosphamide, doxorubicin, vincristine, prednisone; VR-CAP, bortezomib, rituximab, cyclophosphamide, doxorubicin, prednisone.

This analysis supports previous findings of the activity of bortezomib and rituximab-based therapies in this region. ${ }^{32-38}$ The analysis was generally comparable with both IRRC and investigator assessments, with the largest discrepancy between IRRC and investigator assessment for median TTP with VR-CAP (IRRC 30.9 months, investigator assessment 42.7 months).

The safety profiles of VR-CAP and R-CHOP in East Asian patients appeared to generally reflect those reported in the overall LYM-3002 patient population. ${ }^{20}$
All East Asian patients experienced at least one any-grade treatment-emergent $\mathrm{AE}$, irrespective of the treatment they received. Hematologic toxicities were more abundant in the VR-CAP group compared with R-CHOP. This is consistent with previous reports of the increased hematologic toxicity associated with bortezomib. ${ }^{21,35-39}$ Although the incidence of hematologic toxicities was greater in the VR-CAP group, toxicities were effectively managed in both treatment groups by supportive therapies (eg, transfusions, growth factors), as permitted per protocol, and the rates of associated clinically 
Table 4 Most common adverse events (safety population ${ }^{\mathrm{a}}$ )

\begin{tabular}{|c|c|c|c|c|}
\hline \multirow[t]{2}{*}{ Adverse event } & \multicolumn{2}{|c|}{ VR-CAP $(n=72)$} & \multicolumn{2}{|c|}{ R-CHOP $(n=48)$} \\
\hline & Any grade & Grade $\geq 3$ & Any grade & Grade $\geq 3$ \\
\hline Any event, n (\%) & $72(100)$ & $72(100)$ & $48(100)$ & $45(94)$ \\
\hline Any drug-related event, n (\%) & $72(100)$ & $72(100)$ & $47(98)$ & $45(94)$ \\
\hline \multicolumn{5}{|l|}{ Hematologic events, $\mathrm{n}(\%)$} \\
\hline Neutropenia & $72(100)$ & $70(97)$ & $46(96)$ & $38(79)$ \\
\hline Thrombocytopenia & 71 (99) & $60(83)$ & $17(35)$ & $6(13)$ \\
\hline Leukopenia & $64(89)$ & $64(89)$ & $37(77)$ & $26(54)$ \\
\hline Anemia & $58(8 I)$ & $15(21)$ & $28(58)$ & $8(17)$ \\
\hline Lymphopenia & $46(64)$ & $43(60)$ & $20(42)$ & $16(33)$ \\
\hline Febrile neutropenia & $15(2 \mid)$ & $14(19)$ & $9(19)$ & $8(17)$ \\
\hline \multicolumn{5}{|l|}{ Nonhematologic events, n (\%) } \\
\hline Diarrhea & $34(47)$ & $10(14)$ & $5(10)$ & $2(4)$ \\
\hline Pyrexia & $34(47)$ & $5(7)$ & II (23) & $2(4)$ \\
\hline Constipation & $27(38)$ & 0 & $10(21)$ & 0 \\
\hline Fatigue & $27(38)$ & $5(7)$ & $12(25)$ & $2(4)$ \\
\hline Decreased appetite & $24(33)$ & $\mathrm{I}(\mathrm{I})$ & $10(21)$ & I (2) \\
\hline Nausea & $22(3 I)$ & 0 & $4(8)$ & 0 \\
\hline Cough & $19(26)$ & $\mathrm{I}(\mathrm{I})$ & $6(13)$ & 0 \\
\hline Abdominal distension & $15(21)$ & 0 & $\mathrm{I}(2)$ & 0 \\
\hline Peripheral sensory neuropathy & $7(10)$ & $\mathrm{I}(\mathrm{I})$ & $12(25)$ & 0 \\
\hline
\end{tabular}

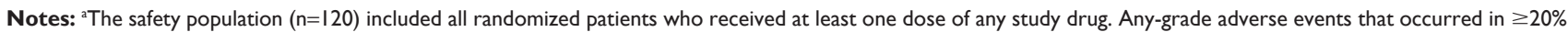
of patients in either arm during the study-treatment period are listed; corresponding rates of grade $\geq 3$ adverse events are also presented. Incidence is based on the number of patients experiencing at least one adverse event.

Abbreviations: R-CHOP, rituximab + cyclophosphamide, doxorubicin, vincristine, prednisone; VR-CAP, bortezomib, rituximab, cyclophosphamide, doxorubicin, prednisone.

significant outcomes were low. There was a slightly higher rate of supportive care used in the East Asian population compared with the overall population $(40 \%$ and $12.5 \%$ compared with $34 \%$ and $18 \%$ for VR-CAP and R-CHOP, respectively). ${ }^{20}$ There was an increased occurrence of febrile neutropenia in the VR-CAP group, which may explain the increased use of colony-stimulating factor in the East Asian population compared with the overall LYM-3002 population. Based on the relative dose intensity, treatment exposure, and discontinuation rates, VR-CAP and R-CHOP appeared as tolerable in East Asian patients as in the overall study population.

Thrombocytopenia is a known reversible, transient, cyclical effect of proteasome inhibition, ${ }^{40}$ rather than a long-term toxic effect. The higher thrombocytopenia rates observed with VR-CAP were reflected in a higher rate of platelet transfusions, but rates of clinically significant bleeding events were similar between arms, indicating no sequelae. Despite the increased rate of anemia with VR-CAP compared with R-CHOP, the number of patients who received an RBC transfusion was similar between the two arms. The percentage of East Asian patients who received an RBC transfusion was lower than in the overall LYM-3002 population in both groups: $15 \%$ and $13 \%$ for VR-CAP and R-CHOP in the East Asian population versus $22 \%$ and $17 \%$ of the overall population. A higher rate of neutropenia in the VR-CAP group was associated with an increase in infections/infestations compared with R-CHOP in East Asian patients (any grade $50 \%$ vs $40 \%$, grade $\geq 325 \%$ vs $19 \%$ ).

Rates of PN were lower in the VR-CAP group than the R-CHOP group (21\% and 29\%). These rates compared favorably with those reported in multiple-myeloma trials $(37 \%-44 \%) .{ }^{41}$ Resolution of clinical neuropathy was similar between groups (VR-CAP 73\%, R-CHOP 79\%). PN is a known reversible side effect of bortezomib, and a known toxicity of vincristine. ${ }^{22,35-37,42-45}$ It seems that the rate of bortezomib/ vincristine-induced $\mathrm{PN}$ was lower in the East Asian population than the overall LYM-3002 population. HBV is prevalent in the East Asian region and reactivation was similar between groups, indicating that the use of VR-CAP over R-CHOP does not increase the risk of HBV reactivation.

A major limitation of this subgroup analysis was the loss of randomization due to the post hoc nature of the analysis. East Asian geography was not considered during patient stratification in the original LYM-3002 study, which led to imbalance in the number of patients in the treatment groups and resulted in some minor differences in patient characteristics, such as median age and rates of bone-marrow involvement.

It should also be noted that while this analysis included all East Asian patients of the LYM-3002 study, the distribution of patients across the East Asian countries (Japan, Republic 
Table 5 Adverse events of clinical interest: thrombocytopenia, neutropenia, and peripheral neuropathy (safety population)

\begin{tabular}{|c|c|c|}
\hline Adverse event & $\begin{array}{l}\text { VR-CAP } \\
(n=72)\end{array}$ & $\begin{array}{l}\text { R-CHOP } \\
(n=48)\end{array}$ \\
\hline \multicolumn{3}{|l|}{ Thrombocytopenia, n (\%) } \\
\hline Any-grade thrombocytopenia & $71(99)$ & $17(35)$ \\
\hline Grade $\geq 3$ thrombocytopenia & $60(83)$ & $6(13)$ \\
\hline Any-grade adverse bleeding events & $2(3)$ & $\mathrm{I}(2)$ \\
\hline Red blood-cell transfusions ${ }^{\mathrm{a}}$ & $11(15)$ & $6(13)$ \\
\hline Platelet transfusions $s^{\mathrm{a}}$ & $25(35)$ & $2(4)$ \\
\hline Cycle delays due to thrombocytopenia & $4(6)$ & $3(6)$ \\
\hline \multicolumn{3}{|l|}{ Neutropenia, n (\%) } \\
\hline Any-grade neutropenia & $72(100)$ & $46(96)$ \\
\hline Grade $\geq 3$ neutropenia & $70(97)$ & $38(79)$ \\
\hline Any-grade adverse infection events & $36(50)$ & $19(40)$ \\
\hline Grade $\geq 3$ adverse infection events & $18(25)$ & $9(19)$ \\
\hline Systemic antibacterial use $\mathrm{a}^{\mathrm{a}}$ & $63(88)$ & $29(60)$ \\
\hline Colony-stimulating factor use $\mathrm{a}^{\mathrm{a}}$ & $96 \% / 26 \%$ & $85 \% / 19 \%$ \\
\hline Reactivation of hepatitis $B$ virus & I (2) & $2(3)$ \\
\hline \multicolumn{3}{|l|}{ Peripheral neuropathy, ${ }^{\mathrm{b}} \mathrm{n}(\%)$} \\
\hline Any-grade peripheral neuropathy & $15(21)$ & $14(29)$ \\
\hline Grade $\geq 3$ peripheral neuropathy & $I(1)$ & I (2) \\
\hline $\begin{array}{l}\text { Treatment discontinuations due to peripheral } \\
\text { neuropathy }\end{array}$ & 0 & 0 \\
\hline \multicolumn{3}{|l|}{ Time to onset of peripheral neuropathy, months } \\
\hline Median & 2.0 & 0.9 \\
\hline Range & $0.6-7.1$ & $0.2-3.9$ \\
\hline $\begin{array}{l}\text { Peripheral neuropathy events improved/ } \\
\text { resolved, } \mathrm{n}(\%)\end{array}$ & $14(93)$ & $12(86)$ \\
\hline Peripheral neuropathy events resolved, n (\%) & II (73) & II (79) \\
\hline \multicolumn{3}{|c|}{ Time to improvement/resolution of peripheral neuropathy, months } \\
\hline Median & 1.9 & 0.6 \\
\hline $95 \% \mathrm{Cl}$ & $0.4-5.6$ & $0.4-7$ \\
\hline \multicolumn{3}{|c|}{ Time to resolution of peripheral neuropathy, months } \\
\hline Median & 11.8 & 8.7 \\
\hline $95 \% \mathrm{Cl}$ & $3-\mathrm{NE}$ & $1.2-11.7$ \\
\hline
\end{tabular}

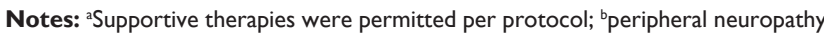
not elsewhere classified, the high-level term including peripheral sensory neuropathy, neuropathy peripheral, peripheral motor neuropathy, and peripheral sensorimotor neuropathy.

Abbreviations: NE, not estimable; R-CHOP, rituximab + cyclophosphamide, doxorubicin, vincristine, prednisone; VR-CAP, bortezomib, rituximab, cyclophosphamide, doxorubicin, prednisone.

of Korea, People's Republic of China, and Taiwan) was not equal. Most patients were of Chinese ethnicity (79\%), with 15\% Japanese, 4\% Korean, and 2\% Taiwanese. Therefore, the results presented here may be more representative of the efficacy and safety of VR-CAP and R-CHOP in Chinese patients.

To conclude, this subgroup analysis of VR-CAP versus $\mathrm{R}-\mathrm{CHOP}$ in East Asian patients with newly diagnosed MCL enrolled in the global LYM-3002 study showed consistent findings with the overall population, with substantially prolonged PFS and improvements in secondary efficacy end points, including $\mathrm{CR} / \mathrm{CRu}$ rates, DOR and $\mathrm{DOCR} / \mathrm{CRu}$,
TTP, TTNT, and TFI. The improved efficacy with VR-CAP relative to R-CHOP was accompanied by increased toxicity; however, this appeared manageable, since this did not influence the number of completed cycles, median dose intensity for drugs common to both regimens, or rates of AE-related discontinuations or deaths (ie, tolerability of VR-CAP compared with R-CHOP was not affected). Tolerability appeared similar in the East Asian population compared with the overall LYM-3002 population. The results suggest that VR-CAP could be considered an effective treatment option for newly diagnosed MCL patients in East Asia for whom intensive treatment and stem-cell transplantation is not an option.

\section{Acknowledgments}

The authors would like to thank the patients who participated in this study and their families, as well as investigators and staff at all LYM-3002 clinical sites. The authors would like to acknowledge Drs Noppadol Siritanaratkul and Goh Yeow Tee for their contributions to the development of this manuscript. Additionally, the authors would like to acknowledge Laura Mulcahy, a medical writer with FireKite, part of Ashfield Healthcare Communications, for writing support during the development of this manuscript, which was funded by Janssen Global Services LLC and Millennium (Takeda Oncology).

\section{Author contributions}

JJ, RO, SSY, LYS, JZ, TL, XNH, and HQH were responsible for study design, JJ, RO, SSY, LYS, JZ, TL, XNH, and HQH patient enrollment, LP, BR, and HV data analysis, and LP, $\mathrm{BR}$, and $\mathrm{HV}$ data interpretation. All authors wrote or contributed to the writing of the manuscript and provided approval of the final manuscript version for submission.

\section{Disclosure}

RO reports grants from Janssen during the conduct of the study. LP reports employment with Janssen R\&D and holding Johnson \& Johnson stocks. BR reports employment with Janssen R\&D and holding Johnson \& Johnson stocks. HV reports employment with Millennium Pharmaceuticals/ Takeda Pharmaceuticals and holding Johnson \& Johnson stocks. The authors report no other conflicts of interest in this work.

\section{References}

1. Herrmann A, Hoster E, Zwingers T, et al. Improvement of overall survival in advanced stage mantle cell lymphoma. J Clin Oncol. 2009; 27(4):511-518.

2. Vose JM. Mantle cell lymphoma: 2013 update on diagnosis, riskstratification, and clinical management. Am J Hematol. 2013;88(12): 1082-1088. 
3. Williams ME. Transplantation for mantle cell lymphoma: is it the right thing to do? Hematology Am Soc Hematol Educ Program. 2013;2013: $568-574$.

4. Lee MY, Tan TD, Feng AC, Liu MC. Clinicopathological analysis of 598 malignant lymphomas in Taiwan: seven-year experience in a single institution. Am J Hematol. 2006;81(8):568-575.

5. Wang JF, Wang YZ, Chen ZW, Taylor RC. [Prevalence of lymphoma subtypes in Shanxi according to latest WHO classification]. Zhonghua Bing Li Xue Za Zhi. 2006;35(4):218-223. Chinese.

6. [No authors listed]. The World Health Organization classification of malignant lymphomas in Japan: incidence of recently recognized entities. Pathol Int. 2000;50(9):696-702.

7. Ko YH, Kim CW, Park CS, et al. REAL classification of malignant lymphomas in the Republic of Korea: incidence of recently recognized entities and changes in clinicopathologic features. Cancer. 1998;83(4): 806-812.

8. Velders GA, Kluin-Nelemans JC, De Boer CJ, et al. Mantle-cell lymphoma: a population-based clinical study. J Clin Oncol. 1996;14(4): 1269-1274.

9. Cancer Research UK. Mantle cell lymphoma. 2014. Available from: http://www.cancerresearchuk.org/about-cancer/type/non-hodgkinslymphoma/about/types/mantle-cell-lymphoma. Accessed May 5, 2016.

10. [No authors listed]. A clinical evaluation of the International Lymphoma Study Group classification of non-Hodgkin's lymphoma. Blood. 1997;89(11):3909-3918.

11. Zhou Y, Wang H, Fang W, et al. Incidence trends of mantle cell lymphoma in the United States between 1992 and 2004. Cancer. 2008; 113(4):791-798.

12. Chuang SS, Huang WT, Hsieh PP, et al. Mantle cell lymphoma in Taiwan: clinicopathological and molecular study of 21 cases including one cyclin D1-negative tumor expressing cyclin D2. Pathol Int. 2006; 56(8):440-448.

13. Howard OM, Gribben JG, Neuberg DS, et al. Rituximab and CHOP induction therapy for newly diagnosed mantle-cell lymphoma: molecular complete responses are not predictive of progression-free survival. J Clin Oncol. 2002;20(5):1288-1294.

14. Ying ZT, Zheng W, Wang XP, et al. The clinical features, therapeutic responses, and prognosis of the patients with mantle cell lymphoma. Chin J Cancer. 2012;31(7):348-353.

15. Miura K, Takasaki H, Tsujimura H, et al. Does more intensive therapy have effects on mantle cell lymphoma? A clinical experience from the Lymphoma Treatment Study Group in Japan. Int J Hematol. 2011;93(5): 684-686.

16. Chihara D, Asano N, Ohmachi K, et al. Prognostic model for mantle cell lymphoma in the rituximab era: a nationwide study in Japan. Br J Haematol. 2015;170(5):657-668.

17. Dreyling M, Thieblemont C, Gallamini A, et al. ESMO consensus conferences: guidelines on malignant lymphoma - part 2: marginal zone lymphoma, mantle cell lymphoma, peripheral T-cell lymphoma. Ann Oncol. 2013;24(4):857-877.

18. McKay P, Leach M, Jackson R, Cook G, Rule S. Guidelines for the investigation and management of mantle cell lymphoma. Br J Haematol. 2012;159(4):405-426.

19. National Comprehensive Cancer Network. NCCN clinical practice guidelines in oncology (NCCN guidelines): non-Hodgkin's lymphomas. Version 4. 2014. Available from: https:/www.nccn.org/about/nhl.pdf. Accessed February 6, 2018.

20. Robak T, Huang H, Jin J, et al. Bortezomib-based therapy for newly diagnosed mantle-cell lymphoma. N Engl J Med. 2015;372(10):944-953.

21. Velcade (bortezomib) for injection: for subcutaneous or intravenous use [prescribing information]. Cambridge (MA): Millennium Pharmaceuticals; 2014

22. Mounier N, Ribrag V, Haioun C, et al. All B lymphoma subtypes do not share similar outcome after frontline R-CHOP plus bortezomib treatment: a randomized phase 2 trial from Groupe d'Etude des Lymphomes de 1'Adulte (GELA). Ann Oncol. 2008;19 Suppl 4:135.
23. He J, Yang L, Han X, et al. The choice of regimens based on bortezomib for patients with newly diagnosed multiple myeloma. PLoS One. 2014;9(6):e99174.

24. Lin M, Hou J, Chen W, et al. Improved response rates with bortezomib in relapsed or refractory multiple myeloma: an observational study in Chinese patients. Adv Ther. 2014;31(10):1082-1094.

25. Wang H, Wang L, Lu Y, et al. Long-term outcomes of different bortezomib-based regimens in Chinese myeloma patients. Onco Targets Ther. 2016;9:587-595.

26. Igarashi N, Chou T, Hirose T, Imai Y, Ishiguro T. Bortezomib and dexamethasone for Japanese patients with relapsed and refractory multiple myeloma: a single center experience. Int J Hematol. 2010;92(3): 518-523.

27. Miyakoshi S, Kami M, Yuji K, et al. Severe pulmonary complications in Japanese patients after bortezomib treatment for refractory multiple myeloma. Blood. 2006;107(9):3492-3494.

28. Gotoh A, Ohyashiki K, Oshimi K, et al. Lung injury associated with bortezomib therapy in relapsed/refractory multiple myeloma in Japan: a questionnaire-based report from the "lung injury by bortezomib" joint committee of the Japanese Society of Hematology and the Japanese Society of Clinical Hematology. Int J Hematol. 2006;84(5):406-412.

29. Venkatakrishnan K, Burgess C, Gupta N, et al. Toward optimum benefit-risk and reduced access lag for cancer drugs in Asia: a global development framework guided by clinical pharmacology principles. Clin Transl Sci. 2016;9(1):9-22.

30. National Cancer Institute. Common terminology criteria for adverse events. 2006. Available from: http://ctep.cancer.gov/protocolDevelopment/electronic_applications/docs/ctcaev3.pdf. Accessed January 19, 2018 .

31. Determann O, Hoster E, Ott G, et al. Ki-67 predicts outcome in advanced-stage mantle cell lymphoma patients treated with anti-CD20 immunochemotherapy: results from randomized trials of the European MCL Network and the German Low Grade Lymphoma Study Group. Blood. 2008;111(4):2385-2387.

32. Flinn IW, van der Jagt R, Kahl BS, et al. Randomized trial of bendamustinerituximab or R-CHOP/R-CVP in first-line treatment of indolent NHL or MCL: the BRIGHT study. Blood. 2014;123(19):2944-2952.

33. Kluin-Nelemans HC, Hoster E, Hermine O, et al. Treatment of older patients with mantle-cell lymphoma. $N$ Engl J Med. 2012;367(6): 520-531.

34. Lenz G, Dreyling M, Hoster E, et al. Immunochemotherapy with rituximab and cyclophosphamide, doxorubicin, vincristine, and prednisone significantly improves response and time to treatment failure, but not long-term outcome in patients with previously untreated mantle cell lymphoma: results of a prospective randomized trial of the German Low Grade Lymphoma Study Group (GLSG). J Clin Oncol. 2005;23(9):1984-1992.

35. Fisher RI, Bernstein SH, Kahl BS, et al. Multicenter phase II study of bortezomib in patients with relapsed or refractory mantle cell lymphoma. J Clin Oncol. 2006;24(30):4867-4874.

36. Goy A, Bernstein SH, Kahl BS, et al. Bortezomib in patients with relapsed or refractory mantle cell lymphoma: updated time-to-event analyses of the multicenter phase 2 PINNACLE study. Ann Oncol. 2009; 20(3):520-525.

37. Kane RC, Dagher R, Farrell A, et al. Bortezomib for the treatment of mantle cell lymphoma. Clin Cancer Res. 2007;13(18 Pt 1):5291-5294.

38. O'Connor OA, Moskowitz C, Portlock C, et al. Patients with chemotherapy-refractory mantle cell lymphoma experience high response rates and identical progression-free survivals compared with patients with relapsed disease following treatment with single agent bortezomib: results of a multicentre Phase 2 clinical trial. Br J Haematol. 2009;145(1): 34-39.

39. Broyl A, Corthals SL, Jongen JL, et al. Mechanisms of peripheral neuropathy associated with bortezomib and vincristine in patients with newly diagnosed multiple myeloma: a prospective analysis of data from the HOVON-65/GMMG-HD4 trial. Lancet Oncol. 2010;11(11): $1057-1065$. 
40. Lonial S, Richardson PG, San MJ, et al. Characterisation of haematological profiles and low risk of thromboembolic events with bortezomib in patients with relapsed multiple myeloma. Br J Haematol. 2008;143(2):222-229.

41. Furman RR, Martin P, Ruan J, et al. Phase 1 trial of bortezomib plus R-CHOP in previously untreated patients with aggressive non-Hodgkin lymphoma. Cancer. 2010;116(23):5432-5439.

42. Goy A, Bernstein SH, McDonald A, et al. Potential biomarkers of bortezomib activity in mantle cell lymphoma from the phase 2 PINNACLE trial. Leuk Lymphoma. 2010;51(7):1269-1277.

43. Hoster E, Klapper W, Hermine O, et al. Confirmation of the mantle-cell lymphoma International Prognostic Index in randomized trials of the European Mantle-Cell Lymphoma Network. J Clin Oncol. 2014;32(13): 1338-1346.
44. Rummel MJ, Niederle N, Maschmeyer G, et al. Bendamustine plus rituximab versus $\mathrm{CHOP}$ plus rituximab as first-line treatment for patients with indolent and mantle-cell lymphomas: an open-label, multicentre, randomised, phase 3 non-inferiority trial. Lancet. 2013;381(9873): 1203-1210.

45. Ribrag V, Gisselbrecht C, Haioun C, et al. Efficacy and toxicity of 2 schedules of frontline rituximab plus cyclophosphamide, doxorubicin, vincristine, and prednisone plus bortezomib in patients with B-cell lymphoma: a randomized phase 2 trial from the French Adult Lymphoma study group (GELA). Cancer. 2009;115(19):4540-4546. 


\section{Supplementary materials LYM-3002 investigators}

Patients were recruited from 23 centers across four countries in East Asia. The following investigators (listed by country) enrolled patients into the study: Japan - Yoshiharu Maeda, Michinori Ogura, Rumiko Okamoto, Masafumi Taniwaki, Yasuhito Terui, Kensei Tobinai, Naokuni Uike, Kiyoshi
Ando, Kenichi Ishizawa, Mitsutoshi Kurosawa, and Akihiro Tomita; China - Huiqiang Huang, Jie Jin, Jun Zhu, Ting Liu, Xiaonan Hong, Xiaoyan Ke, Huaqing Wang, Zhixiang Shen, Yuankai Shi, and Zhao Wang; Republic of Korea - Sung-Soo Yoon and Cheol Won Suh; Singapore - Yeow Tee Goh; and Taiwan - Lee-Yung Shih.

Table SI Institutional review boards (IRBs) and ethics committees (ECs)

\begin{tabular}{|c|c|c|c|}
\hline Country & Name and address & Chairperson(s) & Site \\
\hline \multirow[t]{2}{*}{ China } & NCC Ethics Committee/IRB, I7 Panjiayuan South Road, Beijing I0002I & Datong Chu & 086001 \\
\hline & & & Received drug \\
\hline \multirow[t]{2}{*}{ China } & Beijing Cancer Hospital IEC, 52 Fucheng Road, Haidian, Beijing $100 \mid 42$ & Jie Li & 086002 \\
\hline & & & Received drug \\
\hline \multirow[t]{2}{*}{ China } & Drug Clinical Research IRB of Peking University Third Hospital, 49 North & Xiaoguang Liu & 086003 \\
\hline & Garden Road, Beijing 100083 & & Received drug \\
\hline \multirow[t]{2}{*}{ China } & Tianjin Medical University Cancer Hospital and Institute, Huanhuxi Road, & Ming Gao & 086004 \\
\hline & Tiyuanbei, Tianjin 300060 & Ying Wang & Received drug \\
\hline \multirow[t]{2}{*}{ China } & EC of Rui Jin Hospital, I 97 Ruijin 2 Road, Shanghai 200025 & Su Yan & 086005 \\
\hline & & & Received drug \\
\hline \multirow[t]{2}{*}{ China } & EC of Cancer Hospital, Fudan University, 270 Dong'an Road, Shanghai & Jiong Wu & 086006 \\
\hline & 200032 & & Received drug \\
\hline \multirow[t]{2}{*}{ China } & Sun Yat-sen University Oncology Center, 65I Dongfeng East Road, & Wangqing Peng & 086007 \\
\hline & Guangzhou, Guangdong 510180 & & Received drug \\
\hline \multirow[t]{2}{*}{ China } & First Affiliated Hospital of Zhejiang University of IRB/EC, 79 Qinchun & Kezhou Liu & 086008 \\
\hline & Road, Hangzhou, Zhejiang 310003 & & Received drug \\
\hline \multirow[t]{2}{*}{ China } & EC of West China Hospital, Sichuan University, 37 Guoxuexiang, & Zhi Zeng & 086009 \\
\hline & Wuhou, Chengdu, Sichuan 61004I & & Received drug \\
\hline \multirow[t]{2}{*}{ China } & EC of Beijing Friendship Hospital, 95 Yongan Road, Xuanwu, Beijing & Mei Wei & 086010 \\
\hline & 100050 & & Received drug \\
\hline \multirow[t]{2}{*}{ Japan } & National Hospital Organization Hokkaido Cancer Center Institutional & Hidenori Edo & 081001 \\
\hline & Review Board, 2-3-54 4-jo, Kikushi, Shiroishi-ku, Sapporo 003-0804 & & Received drug \\
\hline \multirow[t]{2}{*}{ Japan } & Tohoku University Hospital Institutional Review Board, I-I Seiryo-cho, & Michiaki Unno & 081002 \\
\hline & Aoba-ku, Sendai $980-8574$ & & Received drug \\
\hline \multirow[t]{2}{*}{ Japan } & Tokyo Metropolitan Cancer and Infectious Diseases Center Komagome & Kazuteru Ohashi & 081003 \\
\hline & Hospital IRB/IEC, 3-I8-22 Honkomagome, Bunkyo-ku, Tokyo I| 3-002I & & Received drug \\
\hline \multirow[t]{8}{*}{ Japan } & Institutional Review Board of National Cancer Center, 5-I-I Tsukiji, & Yoko Ebihara & 081004 \\
\hline & Chuo-ku, Tokyo I04-0045 & Satomi Hirayama & Received drug \\
\hline & & Junko Horie & \\
\hline & & Eiko Mitsumata & \\
\hline & & Chieko Nozawa & \\
\hline & & Yumiko Suzuki & \\
\hline & & Tomohide & \\
\hline & & Tamura & \\
\hline \multirow[t]{6}{*}{ Japan } & Institutional Review Board of National Cancer Center, 5-I-I Tsukiji, & Tomohide & 081005 \\
\hline & Chuo-ku, Tokyo 104-0045 & Tamura & $6-5-1$ \\
\hline & & & Kashiwanoha, \\
\hline & & & Kashiwa, chiba \\
\hline & & & 277-8577 \\
\hline & & & Received drug \\
\hline \multirow[t]{2}{*}{ Japan } & Institute Hospital of JFCR Institutional Review Board, 3-8-3I Ariake, & Masujiro Makita & 081006 \\
\hline & Koto-ku, Tokyo I35-8550 & & Received drug \\
\hline \multirow[t]{2}{*}{ Japan } & Tokai University Institutions Review Board, I43 Shimokasuya, Isehara, & Kiyoshi Ando & 081007 \\
\hline & Kanagawa $259-1193$ & & Received drug \\
\hline \multirow[t]{2}{*}{ Japan } & Nagoya Daini Red Cross Hospital IRB/IEC, 2-9 Myoken-cho, Showa-ku, & Kunio Morozumi & 081008 \\
\hline & Nagoya, Aichi $466-8650$ & & Received drug \\
\hline
\end{tabular}

(Continued) 
Table SI (Continued)

\begin{tabular}{|c|c|c|c|}
\hline Country & Name and address & Chairperson(s) & Site \\
\hline \multirow[t]{2}{*}{ Japan } & Nagoya University Hospital IRB, 65 Tsurumai-cho, Showa-ku, Nagoya, & Toshihiko & 081009 \\
\hline & Aichi $466-8560$ & Wakabayashi & Received drug \\
\hline \multirow[t]{2}{*}{ Japan } & Kyoto Prefectural University of Medicine IRB, 465 Kawaramachi-dori, & Norito Kato & 081010 \\
\hline & Kamigyou-ku, Kyoto $602-8566$ & & Received drug \\
\hline \multirow[t]{2}{*}{ Japan } & National Kyushu Cancer Center Institutional Review Board, 3-I-I & Yasushi To & 081011 \\
\hline & Notame, Minami-ku, Fukuoka 8I I-I395 & & Received drug \\
\hline Republic & Asan Medical Center, IRB of Asan Medical Center, 388-I Pungnap-2- & Jong Woo Chung & 082001 \\
\hline of Korea & dong, Songpa-gu, Seoul I38-040 & & Received drug \\
\hline Republic & Korea Cancer Center Hospital IRB, 75 Nowon-gil, 2 I5-4 Gongneung- & Sang-Moo Lim & 082002 \\
\hline of Korea & dong, Nowon-gu, Seoul I39-706 & & Received drug \\
\hline Republic & Kyungpook National University Hospital IRB, 50 Samduk-2-ga, Jung-gu, & In-Teak Kim & 082003 \\
\hline of Korea & Daegu, North Gyeongsang 700-721 & Jung-Guk Kim & Received drug \\
\hline Republic & Samsung Medical Center IRB, 50 Ilwon-dong, Gangnam-gu, Seoul I35-7I0 & & 082004 \\
\hline \multirow[t]{2}{*}{ of Korea } & & & Did not \\
\hline & & & receive drug \\
\hline \multirow[t]{2}{*}{ Taiwan } & Chang-Gung Memorial Hospital Linkou, 5 Fu-Hsing Street, Kuei Shan & Tsang-Tang & 886001 \\
\hline & Shiang, Taoyuan 333 & Hsieh & Received drug \\
\hline
\end{tabular}

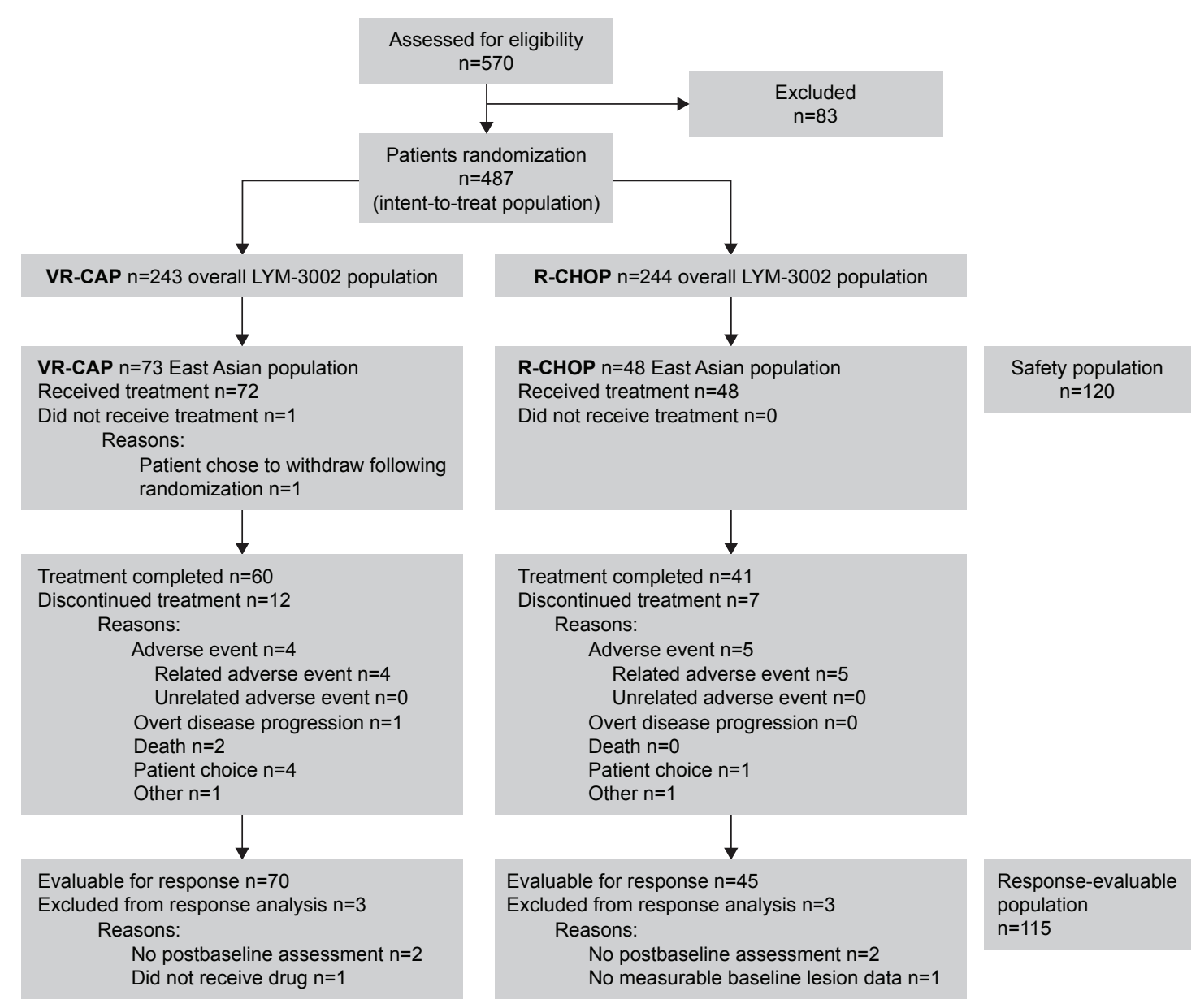

Figure SI Patient disposition (CONSORT diagram).

Abbreviations: VR-CAP, bortezomib, rituximab, cyclophosphamide, doxorubicin, prednisone; R-CHOP, rituximab + cyclophosphamide, doxorubicin, vincristine, prednisone. 


\section{Publish your work in this journal}

OncoTargets and Therapy is an international, peer-reviewed, open access journal focusing on the pathological basis of all cancers, potential targets for therapy and treatment protocols employed to improve the management of cancer patients. The journal also focuses on the impact of management programs and new therapeutic agents and protocols on

patient perspectives such as quality of life, adherence and satisfaction. The manuscript management system is completely online and includes a very quick and fair peer-review system, which is all easy to use. Visit http://www.dovepress.com/testimonials.php to read real quotes from published authors.

Submit your manuscript here: http://www.dovepress.com/oncotargets-and-therapy-journal 